\title{
On An Alleged Non-Equivalence Between Dispositions And Disjunctive Properties
}

\author{
Jonathan Cohen
}

Abstract: This paper shows that grounded dispositions are necessarily coextensive with disjunctive properties. It responds to several objections against this thesis, and then shows how to construct a disjunctive property necessarily coextensive with an arbitrary grounded disposition.

A widely held view about dispositions has it that things have dispositions in some if not all cases in virtue of having some numerically distinct properties, called 'bases' (or 'grounds') of the dispositions. ${ }^{1}$ Let a dispositional property $P$ be called 'grounded' just in case for every instance $x$ of $P, x$ has some or other basis for $P{ }^{2}$ For example, the dispositional property fragility is grounded just in case every fragile thing has some property other than fragility in virtue of which it is fragile.

It seems intuitively plausible that grounded dispositions might be necessarily coextensive (coextensive in all worlds) with some sort of disjunctive properties built from their bases. After all, a dispositional property like fragility applies to a glass in virtue of its having one sort of crystalline structure, to a plastic sheet in virtue of its having another sort of crystalline structure, and so on. Thus, the thought might go, it must be possible to write out some sort of disjunction of such structures that would itself be necessarily coextensive with the disposition fragility. However, Elizabeth Prior has argued that this line of thought is incorrect - that (grounded) dispositions are not necessarily coextensive with disjunctive properties [Prior, 1985]. In this paper I answer Prior's argument, and show that grounded dispositions are necessarily coextensive with disjunctive properties. ${ }^{3}$

\footnotetext{
${ }^{1}$ For present purposes I am allowing that the basis of a disposition may itself be a disposition; cf. [Blackburn, 1990]. For more on dispositions, bases, and related notions, see [McLaughlin, 1995].

${ }^{2}$ Rylean phenomenalism about dispositions (see [Ryle, 1949]) can be understood as the position that not all dispositions need be grounded. Most recent commentators repudiate phenomenalism in favor of the view that all dispositions are grounded (cf. [Armstrong, 1968], 87-88, [Prior, 1985], [Johnston, 1992]). I take no stand on this matter.

${ }^{3}$ Prior argues against a stronger notion of equivalence than the one I am defending: she argues that (grounded) dispositions are not identical to disjunctive properties, while I am only attempting to show that (grounded) dispositions are necessarily coextensive with disjunctive properties. But Prior takes necessary coextension to be a necessary condition for property identity, and argues against the weaker equivalence claim as a way of impugning the stronger equivalence claim. For present purposes I wish to remain agnostic about whether necessary
} 
To begin, consider the grounded dispositional property fragility. ${ }^{4}$ In the actual world $\alpha$ there are many (perhaps infinitely many) low-level configurations that serve as bases for this disposition; let the exhaustive list of such configurations be represented as $P_{1}^{\alpha}, P_{2}^{\alpha}, \ldots$. As Prior points out, fragility is not coextensive with the disjunctive property $\left(P_{1}^{\alpha} \vee P_{2}^{\alpha} \vee \cdots\right)$ even in $\alpha$ ([Prior, 1985], 73; Prior attributes the argument to Frank Jackson in conversation). Prior asks us to imagine that those things of kind $K_{1}$ that have fragility have as their basis $P_{1}^{\alpha}$, while those things of kind $K_{2}$ that have fragility have as their basis $P_{2}^{\alpha}$. But now, she points out, there might be something of kind $K_{1}$ that possesses $P_{2}^{\alpha}$ and lacks $P_{1}^{\alpha}$; this thing will not possess the disposition fragility, although it will possess the disjunctive property $\left(P_{1}^{\alpha} \vee P_{2}^{\alpha} \vee \cdots\right)$. Since this thing will possess the disjunctive property but not the disposition, it is not true that the disposition is coextensive with the disjunctive property.

Luckily, this objection can be answered if we insist that the properties from which we build our disjunctive property (henceforth, 'the disjunct properties') must themselves be conjunctive properties of the form $\left(K_{i} \& P_{i}^{\alpha}\right)$, which apply to only things of kind $K_{i}$ that also have (in $\alpha$ ) the basis $P_{i}^{\alpha}$ for the disposition. Prior is aware of the possibility of this move, but she dismisses it on the grounds that (i) disjunctive properties shouldn't count as genuine properties because they do not have causal powers over and above those of their disjunct properties, (ii) it would have the consequence that 'only in the most artificial sense could one claim that fragility is the same property of the various fragile objects', and (iii) objects having $\left(K_{i} \& P_{i}^{\alpha}\right)$ could fail to be fragile by having an 'internal structural property $S$ which swamped the effect' of the basis ([Prior, 1985], 73).

I'll take these points in turn.

First, (i) is irrelevant to the claim that dispositions are necessarily coextensive with disjunctive properties, even if it were a threat to the stronger claim that the former are identical to the latter: even if a causally potent disposition can't be identified with a causally impotent disjunctive property, there's no reason the two can't be necessarily coextensive. In fact, however, (i) is also unconvincing against the claim that dispositions are identical to disjunctive properties. For it is no less (and no more) controversial that dispositions have causal powers over and above their bases than that disjunctive properties have causal powers over and above their disjunct properties; but since we are taking dispositions to be genuine properties in the present discussion, there is every reason to take the same view of disjunctive properties. ${ }^{5}$

coextension is sufficient for property identity (cf. [Sober, 1982] for an argument that it is not).

${ }^{4}$ Nothing turns on my choice of this example; the reader who thinks fragility is not a grounded disposition is invited to substitute a property that is.

${ }^{5}$ Putting aside the question of whether the causal powers of dispositions (/disjunctive properties) amount to anything over and above those of their bases (/their disjunct properties), some have worried about forging a link between dispositions and disjunctive properties because they think the former are causally potent while the latter are not - i.e., not merely that the latter have causal efficacy over and above the causal efficacy of something else, but that the former but not the latter are causally efficacious at all (see, for example, [Mumford, 1998]).

But this worry seems inconclusive as well. For one thing, it is not uncontroversial that dispositions are causally efficacious (see [Prior et al., 1982]). For another, if dispositions are 
Consider (ii) next. Again, there is no reason that (ii) should block the necessary coextension between an artificially unified disposition and a genuinely unified disjunctive property, even if this were a reason for denying that the two could be identified. However, I don't see that (ii) poses an obstacle to identifying the two either, insofar as it is unclear what it would mean to say that 'fragility is the same property of the various fragile objects' in a non-artificial sense, or why a successful analysis of fragility must ensure this outcome.

I am also unmoved by (iii). For if $S$ swamps $P_{i}$ in object $x$ of kind $K_{i}$, then the basis for the disposition in things of kind $K_{i}$ is not $P_{i}$ but $\left(P_{i} \& \neg S\right)$, so the disjunct property for $x$ is $\left(K_{i} \& P_{i}^{\alpha} \& \neg S\right)$. But this disjunct property is not exemplified by $x$, so $x$ has neither the disjunctive property nor the disposition, and therefore is no obstacle to the coextension of the disjunctive property and the disposition (cf. [Mumford, 1998], 104-5). ${ }^{6} 7$

For these reasons, I think we should feel free to insist that the disjunct properties from which we construct our disjunctive property should themselves be conjunctive properties of the form $\left(K_{i} \& P_{i}^{\alpha}\right)$. On this proposal, then, a dispositional property will be associated with a disjunctive property of the form $\left(K_{1} \& P_{1}^{\alpha}\right) \vee\left(K_{2} \& P_{2}^{\alpha}\right) \vee \cdots$. Of course, we allow that the $P_{i}^{\alpha}$ may themselves be disjunctive properties, insofar as a given kind in $\alpha$ may have distinct possible bases for the given disposition.

But this is still insufficient. For the dispositions a thing has depends crucially on the operative laws of nature: in worlds where the laws of nature are different, none of the $P_{i}^{\alpha}$ need serve as the basis for the disposition fragility. Consequently, we must take into account the (perhaps infinitely many) low-level configurations that serve as the basis for the disposition at each world $w \neq \alpha$ as well; the bases at world $w$ for the disposition, then, let us call $P_{1}^{w}, P_{2}^{w}, \ldots$. Then, corresponding to the disjunctive property above for $\alpha$, we have the disjunctive property $\phi_{w}=$

causally efficacious, it is arguable that their causal efficacy is derivative on that of their bases; but, if so, then we can accord an analogously derivative causal efficacy to disjunctive properties in terms of that of their disjunct properties; and in this case, the worry under consideration reduces to the objection discussed in the main text.

${ }^{6} \mathrm{I}$ 'm assuming that the 'internal structural property' Prior has in mind is to be an intrinsic property of $x$; if the effect of $\left(K_{i} \& P_{i}^{\alpha}\right)$ on $x$ is swamped by the presence of an extrinsic property of $x$, then we should insist that $x$ does indeed have the dispositional property fragility, although the disposition is, in its case, 'masked' — that $x$ indeed has the disposition even though it is prevented from manifesting its disposition by an extrinsic factor (cf. [Johnston, 1992], 146). Thanks to Ron Mallon and Ram Neta for discussion on this point.

${ }^{7}$ Objection (due to Philip Robbins): What if $S$ swamps the disposition in only some instances of $K_{i}$, not all? That is, suppose that $S$ swamps the disposition in $x$ of kind $K_{i}$ but not in $y$ of kind $K_{i}$. Then $y$ would have the disposition for kind $K_{i}$ but lack the disjunctive property.

Reply: In this case we must individuate kinds more finely than we had initially been supposing. In particular, kind $K_{i}$ must be divided into $K_{i_{x}}$ and $K_{i y}$, such that $x$ is a member of kind $K_{i x}$ and $y$ is a member of kind $K_{i y}$. (This kind-refinement seems motivated if, as we are supposing, an intrinsic property shared by $x$ and $y$ swamps the disposition in $x$ but not $y$.) Then the disjunct property for $x$ is $\left(K_{i_{x}} \& P_{i} \& \neg S\right)$ while the disjunct property for $y$ is $\left(K_{i y} \& P_{i}\right)$. This allows us to say that $x$ has neither the disjunctive property nor the disposition, while $y$ has both the disjunctive property and the disposition, which preserves the coextension of the disjunctive property with the disposition. 
$\left(\left(K_{1} \& P_{1}^{w}\right) \vee\left(K_{2} \& P_{2}^{w}\right) \vee \cdots\right)$ for each $w .^{8}$

Finally, we can represent the dispositional property fragility by inserting into the world-bound disjunctive properties $\phi_{w}$ the property $L_{w}$, which will be satisfied by all and only those objects located in world $w$. Doing so, we obtain the disjunctive property

$$
\Phi=\bigvee_{w}\left(L_{w} \& \phi_{w}\right)
$$

I claim having the infinite disjunctive property $\Phi$ is necessary and sufficient for having the grounded disposition (in our case, the disposition fragility). Necessity: if $x$ exemplifies fragility in an arbitrary world $w$, then $x$ must have $L_{w}$; and since, ex hypothesi, the disposition is grounded, there must be some $n$ such that $x$ is of kind $K_{n}$ and a basis $P_{n}^{w}$ (exemplified by $x$ ) for the disposition for objects of kind $K_{n}$ in $w$; this shows that $x$ exemplifies $\left(L_{w} \&\left(K_{n} \& P_{n}^{w}\right)\right)$, so $x$ exemplifies $\left(L_{w} \& \phi_{w}\right)$, so $x$ exemplifies $\Phi$. Sufficiency: if $x$ in $w$ exemplifies $\Phi$, then there is some $n$ such that $x$ exemplifies $\left(L_{w} \&\left(K_{n} \& P_{n}^{w}\right)\right)$; but this means that $x$ is of a kind that, in world $w$, has a property that serves as the basis for the disposition, and hence has the disposition.

Department of Philosophy University of California, San Diego 9500 Gilman Drive

La Jolla, CA 92093-0119 joncohen@ruccs.rutgers.edu

\section{References}

[Armstrong, 1968] Armstrong, D. (1968). A Materialist Theory of the Mind. London: Routledge.

[Blackburn, 1990] Blackburn, S. (1990). Filling in space. Analysis, 50(2):62-65.

[Byrne and Hilbert, 1997] Byrne, A. and Hilbert, D. R., editors (1997). Readings on Color, Volume 1: The Philosophy of Color. MIT Press, Cambridge, Massachusetts.

[Johnston, 1992] Johnston, M. (1992). How to speak of the colors. Philosophical Studies, 68:221-63. Reprinted in [Byrne and Hilbert, 1997], 137-76.

[McLaughlin, 1995] McLaughlin, B. (1995). Disposition. In Kim, J. and Sosa, E., editors, A Companion to Metaphysics, pages 121-24. Blackwell Press, Oxford.

[Mumford, 1998] Mumford, S. (1998). Dispositions. Oxford University Press, New York.

\footnotetext{
${ }^{8} \mathrm{I}$ allow that there may be uncountably many worlds, in which case $w$ will range over an uncountable set.
} 
[Prior, 1985] Prior, E. (1985). Dispositions. Aberdeen University Press, Aberdeen.

[Prior et al., 1982] Prior, E., Pargetter, R., and Jackson, F. (1982). Three theses about dispositions. American Philosophical Quarterly, 19:251-57.

[Ryle, 1949] Ryle, G. (1949). The Concept of Mind. Hutchinson, London.

[Sober, 1982] Sober, E. (1982). Why logically equivalent predicates may pick out different properties. American Philosophical Quarterly, 19:183-89. 